\title{
Das Urteil des Bundesverfassungsgerichts zur Pflegeversicherung
}

\author{
- Grenzen einer ökonomischen Betrachtungsweise
}

\section{Einleitung}

Daß Familien auch in einem reichen Land wie der Bundesrepublik einen schweren Stand haben, wird deutlich, wenn man sich vor Augen hält, daß mehr als is\% der Kinder, das sind mehr als eine Million, Sozialhilfe beziehen.

Worauf ist die relativ ungünstige Position der Familien zurückzuführen? Natürlich haben es Familien in einem marktwirtschaftlichen System schwer, da der Markt Erziehungsleistungen nicht direkt finanziell honoriert, da Erziehung kein marktfähiges Produkt ist, für das eine monetäre Nachfrage besteht. Die immensen Schwierigkeiten bei der Vereinbarkeit von Familien- und Erwerbstätigkeit zeigen auf, mit welchen Problemen Familien im Vergleich zu Alleinstehenden zu kämpfen haben. Übernimmt aber nicht der Sozialstaat die Aufgabe, familiale Leistungen ausreichend zu finanzieren? So soll im Steuerrecht das Existenzminimum der Familie gesichert werden. Es gibt ein breites Spektrum von monetären und realen Transfers des Staates für die Familie, vom Kindergeld über die Wohnungsbauförderung bis hin zum gebührenfreien Studium. Kann man daraus das Fazit ziehen, daß zumindest der Sozialstaat eine Diskriminierung der Institution Familie mit seiner Familienpolitik im Wesentlichen verhindert hat? Betrachtet man aber die einzelnen sozialpolitischen Instrumente, so kann man nicht generell die These vertreten, daß Sozialpolitik per se familienfördernd wirkt und familiale Leistungen der Kindererziehung und -sozialisierung honoriert.

Immer wieder ist darauf hingewiesen worden, daß gerade unsere Sozialversicherung mit ihren Zweigen wenig familienfreundlich ist. Dabei konzentriert sich die Kritik besonders auf die Renten- und Pflegeversicherung. Um zu prüfen, inwieweit dieser Vorwurf der Benachteiligung der Familie berechtigt ist, müssen kurz die wesentlichen Elemente unseres Sozialversicherungssystems dargestellt werden, um dann detailliert auf das Urteil des Ersten Senats des Bundesverfassungsgerichts (BVerfG (200 I b)) zur sozialen Pflegeversicherung einzugehen, der sich die obige Kritik zu eigen gemacht hat.

\section{Die Grundzüge der deutschen Sozialversicherung und ibre familialen Auswirkungen}

Alle unsere Zweige der gesetzlichen Sozialversicherung - wir konzentrieren uns im Folgenden auf die Renten- und später ausschließlich auf die soziale Pflegeversicherung - sind Pflichtversicherungen. Im allgemeinen hat ein Mitglied, das sich im System benachteiligt fühlt, nicht die Option, aus ihm auszutreten. Von daher wiegt

von einer eugenischen Programmierung der eigenen Erbanlagen die »autonome Lebensgestaltung des Einzelnen einschränkt und die grundsätzlich symmetrischen Beziehungen zwischen freien und gleichen Personen unterminiert « (S. 45): Auch wenn dieser Schutz existentieller Unbedingtheit nicht durch »eine ganz normale Abwägung zwischen konkurrierenden Rechtsgütern « relativiert werden dürfe, sei er doch nicht »gegenüber Interessenabwägungen a priori im Recht«(ebd., S. г I6). Welche Rechtsgüter ggf. als höherrangig einzustufen sein können und über die Modalitäten ihres Vorrangs gilt es sich zu verständigen. 
in einer Pflichtversicherung im Vergleich zu einer freiwilligen Versicherung der

Vorwurf der Diskriminierung besonders schwer.

Des weiteren ist unsere Sozialversicherung nach dem Umlageverfahren aufgebaut. Idealiter sollen in jeder Periode die Einnahmen den Ausgaben entsprechen. Im Umlageverfahren findet im Gegensatz zum Kapitaldeckungsverfahren, das wir in der privaten Alterssicherung vorfinden, keine Vermögensansammlung statt. Dennoch erwirbt der Einzelne auch im Umlageverfahren individuelle Versorgungsansprüche durch seine Beiträge. Diese sind in ihrer Höhe einkommensabhängig. Während in der Rentenversicherung die Leistungen im Wesentlichen beitragsbezogen (in Abhängigkeit von der Beitragszeit und der-höhe) sind, gilt dies nicht für die soziale Pflegeversicherung nach dem SGB XI. Die Ansprüche eines Mitglieds bestimmen sich in erster Linie durch seine Pflegebedürftigkeit. Anspruch auf Pflege hat heute jeder Versicherte, wenn er in den letzten zehn Jahren vor der Antragstellung mindestens fünf Jahre in der Pflegeversicherung versichert gewesen ist. Von daher gilt für die Pflegeversicherung nicht das marktwirtschaftliche Prinzip der Äquivalenz, sondern verstärkt das Solidaritätsprinzip. Die Leistungen der Pflegeversicherung sind im Prinzip bedarfsabhängig. Gerade in auf dem Solidaritätsprinzip aufbauenden Systemen sollte dem Gerechtigkeitsaspekt und dem Diskriminierungsverbot ein besonderer Stellenwert zugeordnet werden.

Von daher ist es nicht überraschend, daß wir in der Gesetzlichen Rentenversicherung Umverteilungskomponenten finden, durch die die Stellung der Familie gestärkt werden soll. $\mathrm{Zu}$ denken ist hier u. a. an die Hinterbliebenenversorgung sowie die Anrechnung von Kindererziehungszeiten. Leider findet man solche auf die Familie ausgerichtete Leistungen nicht in der Pflegeversicherung, was aus Gerechtigkeitsgesichtspunkten auf den ersten Blick nicht nachvollziehbar ist und was u. a. Anlaß für eine Klage beim Bundesverfassungsgericht war.

Kernpunkt der Kritik ist dabei, daß allein in der Pflegeversicherung die finanziellen Leistungen (Beiträge) berücksichtigt werden. Hingegen bleiben die realen - Lampert (I 996, 28 I) spricht von naturalen - Leistungen der Familie in der Pflegeversicherung unberücksichtigt. Nun könnte man argumentieren, daß die familialen Leistungen im großen Umfang durch den Familienleistungsausgleich durch den Staat abgegolten worden sind, so daß eine zusätzliche Honorierung der familialen Leistungen in der Pflegeversicherung eine nicht zu rechtfertigende zusätzliche Berücksichtigung darstellen würde. Wie aber Lampert (1996, 32 ff.) aufzeigt, stehen die Leistungen des Staates im Familienleistungsausgleich in keinem Verhältnis zu den Aufwendungen für die Kinder in einer Familie. Man kann den einfachen Schluß ziehen, daß auch im Sozialstaat die Eltern den wesentlichen Anteil der Kosten der Kindererziehung tragen. Dies ist so lange unproblematisch, wie sich die Familie die Erträge der Kindererziehung voll aneignen kann.

Die Kläger gegen das Gesetz über die soziale Pflegeversicherung argumentieren, daß wir eine Diskrepanz haben: Privatisierung der Kosten bei der Familie und Sozialisierung der Erträge in der Sozialversicherung. Diese These wird damit begründet, $\mathrm{da}$ in der Pflegeversicherung, wie in allen Umlageverfahren, die nachwachsende Generation der Kinder die Finanzierbarkeit der Pflegeversicherung sichern. Bei einem festen Beitragssatz führt ein Rückgang der Zahl der Erwerbstätigen zu einer Verringerung des Beitragsaufkommens, so daß die Pflegeversicherung in eine finanzielle Schieflage gerät. Nur eine ausreichende nachwachsende Generation sichert die Pflege von morgen. An diesen familialen Vorleistungen partizipieren aber nicht nur die Familien selbst, sondern auch all die Versicherten ohne Kinder, die keine entsprechenden realen Leistungen erbracht haben. Sie leben so auf Kosten der Familien. 
Diese im Folgenden kritisch zu prüfende Argumentation hat sich das Bundesverfassungsgericht in seinem Urteil im Wesentlichen zu eigen gemacht. Der Kernsatz des Urteils lautet: »Es ist mit Art. 3 I i.V. mit Art. 6 I GG nicht zu vereinbaren, daß Mitglieder der sozialen Pflegeversicherung, die Kinder betreuen und erziehen und damit neben dem Geldbetrag einen generativen Beitrag zur Funktionsfähigkeit eines umlagefinanzierten Sozialversicherungssystems leisten, mit einem gleich hohen Pflegeversicherungsbeitrag wie Mitglieder ohne Kinder belastet werden« (BVerfG (200 I b, I7I 2)).

\section{Thesen zur Urteilsbegründung des Bundesverfassungsgerichts}

Auffallend an der Argumentation des Bundesverfassungsgerichts ist der Tatbestand, daß es sein Urteil sehr ökonomisch-utilitaristisch begründet. Es argumentiert sehr stringent aus einer rein marktwirtschaftlichen Perspektive von Leistung und Gegenleistung. Des Weiteren wird die Familie vom Bundesverfassungsgericht sehr funktionalistisch gesehen, als eine Institution der Sicherung der Pflegeversicherung.

Es soll in diesem Beitrag anhand einiger Thesen aufgezeigt werden, daß

- die ökonomische Sicht des Bundesverfassungsgerichts sehr verengt und zum Teil mit erheblichen Theoriedefiziten behaftet und oft in sich widersprüchlich ist und

- die These, die Nichtberücksichtigung der Betreuung und Erziehung von Kindern bei der Bemessung der Beiträge sei mit Art. 3 Abs. I in Verbindung mit Art. 6 Abs. I GG nicht vereinbar, nicht aufrechterhalten werden kann.

Als pragmatische Schlussfolgerung sollen stattdessen drei Lösungswege als bessere Alternativen zur kinderbezogenen Beitragsdifferenzierung aufgezeigt werden.

These:

Im Generationenvertrag darf man nicht allein die Belastung der Eltern bei der Kindererziebung sehen, sondern muß auch die Versorgungsansprüche der Kinder, die diese als Beitragszahler im Umlageverfahren erwerben, mitberücksichtigen.

Betrachtet man den Familienverband als eine Solidargemeinschaft, so darf der Erste Senat bei seinem Urteil nicht nur die Last der Eltern in Form der Kindererziehung berücksichtigen. Er muß auch die durch ihre Beiträge erworbenen Ansprüche der Kinder im Generationenvertrag mit berücksichtigen. Kinder zahlen ja keine Beiträge im Generationenvertrag aus reinem Pflichtgefühl gegenüber ihren Eltern und erwarten keine Gegenleistung des Sicherungssystems. Primäre Motivation für ihre Beitragszahlung ist vielmehr die Erwartung, mit ihren Beiträgen Leistungsansprüche zu erwerben. Dem Urteil des Bundesverfassungsgerichts liegt eine verengte Zeitabschnittsbetrachtung zu Grunde. Kinder werden einzig und allein als zukünftige Finanziers der Pflegeversicherung angesehen. Kinder finanzieren aber nicht nur während ihres Erwerbslebens die Pflegeleistungen - sowohl ihrer Eltern als auch der Mitglieder ohne Kinder im Umlageverfahren -, sondern sie erwerben mit ihren Beiträgen auch gleichzeitig eigene Ansprüche gegenüber der nachfolgenden Erwerbsgeneration im Generationenvertrag. Würde man in der Pflegeversicherung konsequent das Äquivalenzprinzip anwenden, nach dem die Beiträge zu den marktüblichen langfristigen Zinssätzen verzinst werden, so wäre rein finanztechnisch gesehen der erwartete Nettobarwert von Kindern auch in der umlagefinanzierten Pflegeversicherung gleich Null. Denn das, was sie für die Sicherung der Pflegeleistungen in der Erwerbsphase leisten, genau das fordern sie später zu einer adäquaten Verzinsung wieder ein. 
Anders formuliert: Die Probleme, die Kinder für die nächste Rentnergeneration

lösen, genau die gleichen lasten sie der Enkelgeneration auf. Während in der Rentenversicherung das Äquivalenzprinzip zumindest intragenerativ in Form der Teilhabeäquivalenz erfüllt ist, da sich die Höhe der Rente über die Länge und die Höhe der Beitragszahlungen bestimmt, gilt dies aber nicht für die Pflegeversicherung, in der das Äquivalenzprinzip völlig aufgehoben ist. Bevor man aus Gerechtigkeitserwägungen fordert, zusätzlich eine Familienkomponente einzubauen, wäre zu prüfen, ob man in der Pflegeversicherung auf Umverteilung verzichtet, die u. U. zu ungerechten Belastungen führt, und stattdessen die distributiven Ziele im Steuer- und Transfersystem löst. Auch in der privaten Pflegeversicherung finden wir keine konsequente Anwendung des Äquivalenzprinzips aufgrund gesetzlicher Vorgaben. Z. B. ist u.a. eine Staffelung der Prämien nach Geschlecht und Pflegeanfälligkeit nicht zugelassen.

\section{These:}

Je geringer die Kosten der Kindererziehung sind bzw. je stärker diese von der Gesellschaft, insbesondere von den Kinderlosen, über Stewern gedeckt werden, um so geringer ist die Ungerechtigkeit einheitlicher Beitragssätze.

Wenn man eine Differenzierung der Beitragssätze zur Pflegeversicherung fordert, so muß man erst einmal detailliert prüfen, welche Lasten der Kinderaufzucht die Gesellschaft und welche die Eltern tragen. Je geringer die Kosten der Kindererziehung für die Eltern sind bzw. je stärker diese von der Gesellschaft, insbesondere von den Kinderlosen, über Steuern getragen werden, um so geringer ist die Ungerechtigkeit einheitlicher Pflegesätze in der Pflegeversicherung.

Wenn die Gesellschaft die Kosten der Kinderziehung in ausreichendem Maße übernimmt, so daß die Eltern keine übermäßigen Kosten für ihre Kinder aufgebürdet bekommen und so keine unzumutbaren Vorleistungen für die finanzielle Stabilität eines Umlagesystems erbringen müssen, dann besteht auch keine besondere gesellschaftliche Verpflichtung, familiale Erziehungsleistungen in der Pflegeversicherung $\mathrm{zu}$ honorieren. Daß die Leistungen der Gesellschaft für die Familie im Familienleistungsausgleich zu niedrig sind, ist politisch zu bewerten. Nach Ott (200I, 263), die sich auf das neue Gutachten des Wissenschaftlichen Beirates beim Bundesministerium für Familie, Senioren, Frauen und Jugend beruft, werden nur 35\% bis $50 \%$ der Kosten von der Gesellschaft getragen. Nach Berechnungen des Sachverständigenrates (200I, Ziff. 274) belaufen sich die staatlichen Familienleistungen auf I48,8 Mrd. Euro pro Jahr und decken so $50 \%$ der Kosten der Kindererziehung ab. Wichtig für das Urteil des Bundesverfassungsgerichts ist hier allein der Sachverhalt, daß nicht allein eine Option - wie vom Senat behauptet -, sondern mindestens zwei Optionen zur Honorierung familialer Leistungen bestehen. Es ist nicht zwingend, über eine Beitragsdifferenzierung Familien in der Pflegeversicherung besser zu stellen, wie dies der Erste Senat kategorisch fordert. Es muß dem Gesetzgeber offen stehen, wie er familiale Leistungen adäquat honoriert. Dazu kann er auch auf eine Verbesserung des Familienleistungsausgleichs zurückgreifen. Es ist nicht zwingend, die Besserstellung der familialen Leistungen an den Beitragssätzen der Pflegeversicherung festzumachen.

\section{These:}

Die funktionalistische, finanztechnische Betrachtungsweise des Bundesverfassungsgerichts wird der realen Leistung der Familie nicht gerecht. 
Des weiteren ist die Begründung einer Beitragsdifferenzierung seitens des Bundesverfassungsgerichts mit dem Hinweis auf »systemspezifische« Vorteile für kinderlose Pflegeversicherte recht problematisch. Mit Recht betont das Bundesverfassungsgericht (200 I b, I7 I 5) unter Bezug auf sein Urteil von I 988 zur Rentenversicherung, daß aus der allgemeinen Angewiesenheit auf die Wertschöpfung durch die heranwachsende Generation nicht abzuleiten ist, die Erziehungsleistung besonders in der Pflegeversicherung zu berücksichtigen. Diese Notwendigkeit ergäbe sich hingegen aus der besonderen Finanzierung der Pflegeversicherung, die nur mit der nachwachsenden Generation gesichert sei. »Die kindererziehenden Versicherten sichern die Funktionsfähigkeit der Pflegeversicherung also nicht nur durch Beitragszahlung, sondern auch durch Betreuung und Erziehung von Kindern « (BVerfG 200 I b, I7 I 5). Daß für das Bundesverfassungsgericht die Finanzierungsform ausschlaggebend ist, wird besonders in seinem Urteil zur »Verfassungsmäßigkeit der privaten Pflegeversicherung « deutlich. Nach Ansicht des Bundesverfassungsgerichts (200I a, I709) ist die private Pflegeversicherung in Form der Anwartschaftsdeckung im wesentlichen kapitalgedeckt »und nicht in gleicher Weise auf die Prämienzahlungen der nachwachsenden Generationen angewiesen, wie die soziale Pflegeversicherung «. Erst wenn sich die Umlagekomponente durchsetzt, stellt sich die Frage einer entsprechenden Beitragsdifferenzierung.

Wenn für das Bundesverfassungsgericht das Finanzierungsargument für die zwingende Beitragsdifferenzierung in der sozialen Pflegeversicherung ausschlaggebend ist, so könnte die Bundesregierung dadurch, daß sie die implizite Schuld des Umlageverfahrens in eine explizite umwandelt, der Argumentation des Bundesverfassungsgerichts die Grundlage entziehen. Daß dies eine durchaus reale Option darstellt, zeigt der Einstieg in das Kapitaldeckungsverfahren bei der Rentenversicherung.

In der Pflegeversicherung erwerben die Versicherten letztlich finanzielle Ansprüche in Abhängigkeit von ihrem Pflegebedarf. Der Staat könnte diese Ansprüche der Versicherten z. B. durch die Emission von Schuldverschreibungen an die Versicherten abdecken und damit die Finanzierung im Umlageverfahren formal in ein kapitalgedecktes System transformieren, wie dies z. B. für die Rentenversicherung Föhl und Buchanan gefordert haben und im Prinzip in Chile realisiert worden ist. Durch den Wechsel wären die systemspezifischen Tatbestände beseitigt, die für die Argumentation des Bundesverfassungsgerichts ausschlaggebend waren. Da eine kapitalgedeckte Pflegeversicherung vorliegt, müßte man - macht man sich die Argumentation des Bundesverfassungsgerichts zu eigen - die familiale Leistung nicht mehr in der Pflegeversicherung berücksichtigen.

Weist man die hier vorgebrachte Argumentation als eine rein finanztechnische Betrachtungsweise zurück und argumentiert, daß sich ja durch die Transformation der impliziten in die explizite Schuld die familiale Leistung für die Pflegeversicherung nicht geändert hat, dann muß das Bundesverfassungsgericht sich die Kritik des Sozialbeirats (200I, Ziff. I I) zu eigen machen, der darauf hinweist, daß eine Differenzierung zwischen impliziter und expliziter Schuld problematisch ist. Wenn aber die Pro-Kopf-Staatsverschuldung von der Bevölkerungsentwicklung und damit ebenfalls von den familialen Leistungen abhängt, warum wird dann dieser Aspekt nicht berücksichtigt? Man kann aus diesen Überlegungen nur den Schluß ziehen, daß die rein finanztechnische Betrachtungsweise des Bundesverfassungsgerichts, die die Relevanz familialer Leistungen an die Existenz des Umlageverfahrens koppelt, den realen Leistungen der Familie nicht gerecht wird. 
Eine funktionale Zuordnung von familialen Leistungen auf einzelne gesellschaftliche Subsysteme und ibre Honorierung in denselben ist aufgrund wechselseitiger Interdependenzen zwischen ibnen weder sinnvoll noch machbar.

Für die Frage, ob und wie familiale Leistungen honoriert werden sollen, ist es letztlich irrelevant, ob ein Kapitaldeckungs- oder ein Umlageverfahren vorliegt. Kindererziehung ist eine gesellschaftliche Aufgabe, die auch in einem rein marktlichen System ohne jegliche staatliche soziale Absicherung zu erfüllen ist. Eine funktionale $\mathrm{Zu}$ ordnung auf einzelne gesellschaftliche Subsysteme und ihre Abgeltung in ihnen ist aufgrund wechselseitiger Interdependenzen der Subsysteme weder sinnvoll noch machbar.

Dies wird besonders deutlich, wenn man die Determinanten der für die Finanzierung der Pflegeversicherung strategischen Größe, der Lohnsumme, betrachtet. Die Lohnsumme als Produkt von Lohn und Beschäftigung hängt nicht direkt von der Bevölkerungsentwicklung ab, die für das Bundesverfassungsgericht die strategische Komponente darstellt. Bei dieser Beziehung zwischen Bevölkerungsentwicklung und Lohnsumme treten u.a. als intervenierende Variable die Erwerbsquote und die Arbeitslosenquote auf. Nun sind aber die Erwerbs- und Beschäftigungsquoten (noch wichtiger die Lebenseinkommen) der Frauen systematisch niedriger als die der Männer. Bei der funktionalistischen Sicht des Bundesverfassungsgerichts müßte dies bei einer typisierenden Betrachtungsweise zu der allen Gerechtigkeitsüberlegungen widersprechenden Forderung führen, eine Differenzierung der Beitragssätze nach dem Geschlecht der Kinder vorzunehmen, da der erwartete Beitrag von männlichen Kindern höher als der weiblichen sein wird.

Aus der funktionalistischen Perspektive müßte das Bundesverfassungsgericht sich eigentlich auch für eine Beitragsdifferenzierung in Abhängigkeit vom Einkommen der Eltern aussprechen, so daß Eltern mit höherem Einkommen geringere Beitragssätze haben. Denn empirische Analysen zeigen, daß die Kinder von erfolgreichen Eltern mit entsprechend hohem Einkommen als Erwachsene mit relativ großer Wahrscheinlichkeit zu den Personen mit hohem Einkommen zählen. ${ }^{\text {I }}$ So nehmen nach der I6. Studie »Zur wirtschaftlichen Lage der Studierenden in der Bundesrepublik Deutschland « nur I $2 \%$ der Arbeiterkinder, aber 75\% der Beamtenkinder und $60 \%$ der Kinder von Selbständigen und Freiberuflern ein Hochschulstudium auf. Bei einer typisierenden Betrachtung wäre deshalb eine solche Differenzierung angebracht.

Betrachtet man die zweite Determinante der Lohnsumme, den durchschnittlichen Lohnsatz, so wird dieser von einer Vielzahl von ökonomischen Faktoren beeinflußt:

$W=W(A, K, H K, H V, F u E)$.

In der Argumentation des Bundesverfassungsgerichts wird nur ein einziger Bestimmungsfaktor indirekt über die Bevölkerungsentwicklung berücksichtigt: der Faktor Arbeit $(A)$. Die anderen und mindestens genauso wichtigen Faktoren wie Realkapital $K$, Humankapital $H K$, Humanvermögen $H V$ und die Forschungs- und Entwicklungsaktivitäten $F u E$ in einer Volkswirtschaft, die entscheidenden Einfluß auf die Lohnsumme haben, bleiben unerklärlicher Weise in der Analyse des Bundesverfassungsgerichts unberücksichtigt.

Steigt das Arbeitsangebot aufgrund des starken Bevölkerungswachstums, so bewirkt dies, daß bei einem normalen Verlauf der Arbeitsnachfragefunktion der Unterneh- 
men der durchschnittliche Lohnsatz sinkt. Wir erhalten so zwei gegenläufige Einflüsse auf die Lohnsumme: Beschäftigungszunahme und Lohnsenkung. Welcher Einfluß der stärkere ist, hängt von der Nachfrageelastizität ab.

Insbesondere dann kann sich ein starkes Bevölkerungswachstum negativ auf die Wachstumsrate des Pro-Kopf-Einkommens und so auf die finanzielle Stabilität eines Umlageverfahrens auswirken, wenn - wie in vielen Entwicklungsländern gegeben die Arbeitsintensität $A / K$ aufgrund einer unzureichenden Kapitalausstattung hoch ist. Sollte man dann kinderreiche Familien wie China einfach abstrafen?

Nun zeigen aber wachstumstheoretische Überlegungen, daß für die Entwicklung der Lohnsumme Faktoren wie das Humankapital und das Humanvermögen sowie Forschungs- und Entwicklungsaktivitäten viel entscheidender als die Bevölkerungsentwicklung selbst sind. Während man unter Humankapital die Bildungsinvestitionen versteht, die sich direkt in einer höheren Arbeitsproduktivität niederschlagen, ist die Konzeption des Humanvermögens, wie sie im Fünften Familienbericht (I994) ausführlich dargestellt ist, viel umfassender. Humanvermögen, das im Wesentlichen durch familiale Leistungen zustande kommt, beinhaltet Daseinskompetenz, Lernfähigkeit, Verantwortungsbewußtsein und Kooperationsbereitschaft. Wäre es dann z. B. aus der Sicht des Bundesverfassungsgerichts nicht konsequent, wenn man den Familien besonders niedrige Beitragssätze gewähren würde, die nicht nur Kinder aufziehen, sondern die diese auch mit viel Humankapital und -vermögen ausstatten und dann noch viel Realkapital vererben?

Diese Schlußfolgerungen machen deutlich, daß das Bundesverfassungsgericht klugerweise nicht bereit ist, seine ökonomistische Betrachtung familialer Leistungen konsequent durchzuhalten.

Darüber hinaus ist zu fragen, warum das Bundesverfassungsgericht nicht verlangt, daß Eltern in der privaten Pflegeversicherung einen Anspruch auf Beitragsdifferenzierung zuzugestehen ist, hängt doch auch in einer privaten Pflegeversicherung die finanzielle Solidität vom reproduktiven Verhalten ab, wenn es um ausreichendes und nicht zu teures Pflegepersonal geht und wenn insbesondere die privaten Pflegeversicherungen nur unzureichend kapitalgedeckt sind. ${ }^{2}$ Des weiteren finanzieren zum überwiegenden Teil die Kinder von privat versicherten Eltern später als Mitglieder der sozialen Pflegeversicherung ein umlagefinanziertes System, woraus ihre Eltern entsprechende Ansprüche ableiten könnten. Und wie sollen die Eltern berücksichtigt werden, die überhaupt keiner Pflegeversicherung angehören?3

These:

Die vorbersebbaren langfristigen Finanzierungsprobleme aufgrund des Geburtenrückganges sind für die Frage der Honorierung familialer Leistungen in der Pflegeversicherung irrelevant.

Besonders betont das Bundesverfassungsgericht (200 I b, I I I 5 ) zu seiner Begründung einer Beitragsdifferenzierung die vorhersehbaren langfristigen Finanzierungsprobleme aufgrund des Geburtenrückganges. Dabei zeigt es ungewöhnlich ausführlich auf, daß der dramatische Geburtenrückgang vorhersehbar war und in absehbarer Zeit nicht revidierbar sei. Es müßte - so das Ergebnis der Anhörungen - die Geburtenrate von I,3 auf 3,8 ansteigen. Dies ist eine völlig unrealistische Steigerungsrate. Auch durch Migration kann die Entwicklung zu steigenden Beitragssätzen bei annähernd

2 Vgl. die entsprechende Kritik von Borchert (200I, 256) am Urteil des BVerfG.

3 Entsprechend weist Rürup (200I, 260) auf die damit einhergehende "Diskriminierung von kinderauf- und erziehenden Beamten, Richtern, Freiberuflern, Selbständigen, Arbeitslosen oder Sozialhilfeempfängern« hin. 
konstantem Leistungsniveau nicht gestoppt werden, da dies bis 2050 eine Zuwanderung von ca. I 88 Mio. Personen voraussetzen würde.

Diese Ausführungen zu den gravierenden Zukunftsproblemen der sozialen Pflegeversicherung und der zu befürchtenden enormen Beitragssatzsteigerung sind aber irrelevant für die funktionale Argumentation des Bundesverfassungsgerichts. Die Frage der Diskriminierung kindererziehender gegenüber kinderlosen Beitragszahlern ist völlig unabhängig von der zu erwartenden Höhe des Beitragssatzes. Mit Recht weist das Bundesverfassungsgericht darauf hin, daß die Benachteiligung der Familie einen intragenerativen Konflikt zwischen denjenigen, die Kinder erziehen, und den kinderlosen Mitgliedern der sozialen Pflegeversicherung darstellt. Die Notwendigkeit der Beitragsdifferenzierung ist nach seiner Ansicht eine Konsequenz der Dualisierung der Gesellschaft in die Klasse der Mitglieder mit Kindern und ohne.

Ironisch könnte man aus diesen Überlegungen z. B. folgende Frage ableiten: Wenn die Reproduktionsrate bei einem Teil der Bevölkerung dramatisch ansteigen würde und eine ausreichende kompensierende Migration realisierbar wäre, so daß die finanzielle Stabilität der Pflegeversicherung gesichert wäre, stellt sich dann nicht mehr die Frage der Benachteiligung der Mitglieder mit Kindern? Wesentliche Ursache für den Geburtenrückgang ist für das Bundesverfassungsgericht (200 I b, I I I s) die Zunahme der kinderlosen Mitglieder, die nicht mehr »in der deutlichen Minderheit « sind. Es wird von ihm unterstellt, daß letztlich immer mehr Kinderlose auf Kosten der Familien ihre Pflege sichern. Mit Recht weist Ruland (200I, I674) mit entsprechenden empirischen Belegen darauf hin, daß in erster Linie der Rückgang der Geburtenrate auf die gesunkene Kinderzahl in den Familien zurückzuführen ist. Aus der funktionalen Perspektive müßten nicht in erster Linie Kinderlose, sondern primär Familien mit zu wenig Kindern Adressat des Urteils des Bundesverfassungsgerichts sein, da diese Familien den Geburtenrückgang und damit die zukünftige finanzielle Instabilität der Pflegeversicherung zu verantworten haben.

Des weiteren kann der Erste Senat nicht ernsthaft die Auffassung vertreten, daß, wenn rechtzeitig 1994 die Benachteiligung der Mitglieder mit Kindern ausgeräumt worden wäre, es dann nicht zu dem schwerwiegenden Bevölkerungsrückgang gekommen wäre. Es wäre naiv anzunehmen, daß Beitragsvariationen das Reproduktionsverhalten grundlegend beeinflussen. Die Kernfrage, die sich bei seinen Ausführungen stellt, lautet, wenn eine Honorierung familialer Leistungen bei ausreichender Reproduktionsrate und adäquater Migration funktional für die finanzielle Stabilität der Pflegeversicherung nicht notwendig wäre, ist damit auch die elementare Forderung gegenstandslos, daß allein aufgrund der Artikel 3 Abs. I und Artikel 6 Abs. I GG ein Handlungsbedarf zur Honorierung der Erziehungsleistungen besteht? Akzeptiert man diesen, dann ist es wenig sinnvoll, die Honorierung familialer Leistungen zwingend in der Pflegeversicherung durch Beitragsdifferenzierung zu verwirklichen.

\section{These:}

Bei der Sozialisierungsthese familialer Leistungen im System der umlagefinanzierten Alters- und Pflegeversicherung wird nicht ausreichend zwischen den unvollständigen Eigentumsrechten im Innenverbältnis und den externen Effekten im Außenverbältnis der Familie differenziert.

Eine weitere Schwäche in der Begründung seines Urteils liegt darin, daß das Bundesverfassungsgericht nicht ausreichend zwischen den unvollständigen Eigentumsrechten im Innenverhältnis und den externen Effekten im Außenverhältnis der Familie differenziert. Das Umlageverfahren ist das älteste und bewährteste System der Alters- 
sicherung und bis zum Beginn der Industrialisierung ohne Alternative für den Großteil der Gesellschaft gewesen, während das Kapitaldeckungsverfahren als umfassendes Alterssicherungs- und Pflegesystem noch seine Bewährungsprobe bestehen muß. Das Umlageverfahren war über Jahrtausende deshalb konkurrenzlos, da die Vorleistungen der Eltern für ihre Kinder bei der Aufzucht relativ gering waren. Kinder waren in den familialen Produktionsprozeß relativ schnell integriert. Die Bildung von Humankapital und -vermögen war relativ bedeutungslos bzw. ließ sich ohne großen Aufwand realisieren. Des Weiteren war das Alterssicherungsniveau extrem niedrig. Man arbeitete im Prinzip bis zum Ende des Lebens, und die Lebenserwartung war niedrig. Darüber hinaus war die Erfüllung des Generationenvertrages im Familienverband gesichert.

Hinzu kam die geringe Mobilität der Familienangehörigen. Die Option, sich durch Abwanderung seiner familialen Pflichten zu entziehen, stand im allgemeinen nicht zur Verfügung bzw. war für viele die ultima ratio. Neben der räumlichen Bindung sorgte die soziale Kontrolle für die Erhaltung des Generationenvertrages.

Die Entwicklung des Kapitalismus schaffte aber eine völlig neue Alternative der Alterssicherung in Form der Kapitalakkumulation. Zum einen führte die gestiegene Produktivität dazu, daß man zum Teil über Einkommen verfügte, das das Existenzminimum überstieg, so daß man für seine Alterssicherung Realkapital akkumulieren konnte. Zum anderen entstand ein funktionsfähiger Kapitalmarkt, der die Eigentumsansprüche der Kapitalgeber ausreichend schützte. Während Investitionen in die eigenen Kinder unvollkommene Eigentumsrechte für die Alterssicherung darstellen, bot sich mit der Kapitalakkumulation nun eine Chance, umfassend abgesicherte Versorgungsansprüche zu erwerben.

Aus dieser Sicht sind heute Kinder keine attraktive Form der familialen Alterssicherung, da keine ausreichende Amortisation elterlicher Vorleistungen garantiert ist, da Kinder diese nicht mehr per se honorieren. Kernproblem unzureichender elterlicher Vorleistungen bei der Erziehung von Kindern ist die unzureichende Solidarität in der Familie. Wäre sie z. B. durch familialen Altruismus gekennzeichnet, so kämen nach Barro (1974) in der Familie nur effiziente intertemporale Entscheidungen zustande, und eine Ausdifferenzierung der Gesellschaft in die Mitglieder der Pflegeversicherung mit Kindern und solche ohne wäre im Prinzip gegenstandslos.

Oft wird die These vertreten, daß die Institution der staatlichen Sozialversicherung Ursache für familiale Ineffizienzen sei, insbesondere die niedrige Geburtenrate zur Folge habe. ${ }^{4}$ Diese These findet man auch im Urteil des Bundesverfassungsgerichtes, das auf die unzumutbar hohen Beitragssätze für kinderreiche Mitglieder der Pflegeversicherung hinweist.

Dem wird hier die These entgegengestellt, daß entscheidender Grund für die Entstehung der staatlichen und auch der genossenschaftlichen sozialen Einrichtungen Defizite in der Familie selbst sind, die keine ausreichend große Risikogemeinschaft darstellt und in der keine ausreichende Solidarität existiert.

In der politischen Diskussion um die Alterssicherung und die Pflegeversicherung stehen nicht die intrafamilialen Konflikte im Vordergrund, sondern die externen Effekte, die von familialen Leistungen für die Kinder zugunsten der Gesellschaft ausgehen. Unter externen Effekten versteht man hier die positiven Auswirkungen auf den Wohlstand einer Gesellschaft, die durch die Kindererziehung bewirkt werden. Engagieren sich Eltern für ihre Kinder, so partizipieren davon auch andere Gesell-

4 Diese Argumentation wird besonders von Kohli/Künemund/Motel/Szydlik (1999) kritisiert, die aufzeigen, daß der Wohlfahrtsstaat erst die materiellen Voraussetzungen für solidarisches Verhalten in der Familie geschaffen hat. 
schaftsmitglieder, ohne daß dies von den Eltern direkt beabsichtigt ist. Man spricht so von der Sozialisierung familialer Leistungen. Plakativ formuliert besagt dies: »Die Familie trägt die Kosten der Kindererziehung, und die Gesellschaft eignet sich die Erträge an.«

Diese These kann aber nicht in dieser undifferenzierten Form akzeptiert werden. Leistungen der Eltern für ihre Kinder sind im Allgemeinen keine Leistungen, die von der Gesellschaft ohne weiteres vereinnahmt werden können. Z. B. erhöhen familiale Humankapitalinvestitionen in die eigenen Kinder deren individuelle Produktivität, die so ein höheres Markteinkommen erzielen können. Natürlich kann die Gesellschaft z. B. über die Besteuerung des Arbeitseinkommens die Erträge aus familialen Vorleistungen sozialisieren. Dies ist aber ein steuerrechtliches und kein sozialversicherungsrechtliches Problem. Gesellschaftliche externe Effekte sind durchaus gegeben, wenn es um die familiale Humanvermögensbildung geht. Hier treten durchaus nennenswerte Externalitäten auf. Kooperative Einstellungen, Ehrlichkeit, Vertragstreue usw. bewirken positive Wohlfahrtseffekte nicht nur bei dem, der über diese Verhaltenseinstellungen verfügt, sondern auch bei denen, die mit ihm interagieren. Die Rendite aus Humanvermögen ist um so größer, je mehr Personen darüber verfügen.

Externe gesellschaftliche Effekte werden aber auch durch die Pflegeversicherung selbst generiert. Dabei sind aber die Zusammenhänge zwischen der Kinderzahl und dem externen Effekt der finanziellen Stabilität der Pflegeversicherung wesentlich komplexer, wie insbesondere die Ausführungen zu den Bestimmungsfaktoren der Lohnsumme zeigen sollten.

\section{Zur adäquaten Honorierung familialer Erziehungsleistungen}

Wenn man akzeptiert, daß das Kernproblem im intrafamilialen Spannungsfeld der unzureichenden Honorierung elterlicher Erziehungsleistungen durch die Kinder liegt, so müssen Wege der Internalisierung aufgezeigt werden. Es muß dafür gesorgt werden, daß Kindererziehung sich auch für Eltern lohnt, die nicht so altruistisch sind, wie wir uns dies wünschen.

Zwei Alternativen stehen zur Diskussion: einmal das Kreditmodell von Schreiber (1955) und das der Elterndividende von Burggraf (1997). Nach Schreiber ist es nicht möglich, aufgrund der Unvollkommenheit des Kapitalmarktes, daß die Kinder ihre eigene Erziehung selbst über den Kapitalmarkt finanzieren. Dies hat zur Folge, daß die Eltern die Finanzierungsfunktion übernehmen, ohne daß der Kredit der Eltern ausreichend abgesichert ist, was zu den oben aufgezeigten intrafamilialen Spannungen führt. Schreiber schlägt nun vor, daß der Staat anstelle der Eltern die Aufgabe des Finanziers übernimmt. Wenn die Kinder erwachsen sind, zahlen sie ihren Kredit zurück. Mit den zurückgezahlten Krediten wird dann das Humankapital der nachfolgenden Generation von Kindern finanziert. Der wesentliche Vorteil dieses Verfahrens liegt darin, daß nun die Kinder ihren Verpflichtungen nachkommen müssen und die finanziellen Vorleistungen auch später honorieren müssen. Denn der Staat verfügt über ein ausreichendes Machtpotential, die Kinder zur Vertragserfüllung zu zwingen. Dieser Vorschlag ist aber nicht unproblematisch. Verträge sollen im Einvernehmen von freien selbstbestimmten Vertragspartnern abgeschlossen werden. Kinder haben aber nicht diesen Status, so daß beim Schreiberplan der Staat einseitig die Konditionen für die nachwachsende Generation diktieren kann. Durch Mitspracherechte der Eltern als Treuhänder der Kinder kann aber dieser Konflikt entschärft werden. Ein weiteres Problem liegt in der Mobilität. Wenn die Kinder später als Erwerbspersonen ihr Heimatland verlassen, dann kann es schwierig - 
wenn nicht gar unmöglich - sein, die staatlichen Ansprüche im Ausland durchzusetzen.

Ähnliche Probleme tauchen bei dem anderen Vorschlag der Elterndividende von Burggraf (I 997, 69 ff.) auf. Sie lehnt eine Vergesellschaftung der Finanzierung ab. Für sie sollen die Eltern weiter die Kosten der Kindererziehung tragen. Sie möchte nur die Eigentumsrechte an den Erträgen der Kindererziehung vervollständigen. Dafür schlägt sie eine Familiensteuer vor. Diese Steuer, die die Kinder zu zahlen haben, fließt nicht an den Staat, sondern direkt an die Eltern. Durch diese Steuer erwerben so die Eltern einen Anteil an der Rendite ihrer Kindererziehung.

$\mathrm{Daß}$ dieser Vorschlag von Burggraf zumindest ansatzweise realisierbar ist, zeigen die Regelungen des Elternunterhalts ( $\int \mathbb{S}$ I60I ff. BGB) sowie des gesetzlichen Forderungsübergangs auf den Sozialhilfeträger ( $\$$ 9I BSHG), welche eine Leistungsverpflichtung der Kinder gegenüber ihren Eltern in bestimmten Notsituationen bedeuten. Vergleicht man beide Lösungsansätze, so spricht vieles für den Schreiberplan, da das Konzept der Elterndividende eine extrem starke Verrechtlichung familialer Beziehungen beinhaltet und der altruistischen Einstellung in der Familie nicht besonders förderlich sein wird. Sollte man z.B. den Eltern über die Elterndividende Anreize geben, primär die Kinder, die die höchste Humankapitalrendite erwarten lassen, zu fördern, oder ist es nicht die Aufgabe der Eltern, die besonders schwachen Kinder zu fördern? Liebe kann man nun mal nur partiell durch Geld und finanzielle Anreize ersetzen.

Bei beiden Vorschlägen geht es aber nur um familieninterne externe Effekte. Ungelöst sind aber die ebenfalls existierenden Externalitäten auf der gesellschaftlichen Ebene. Diese sind wesentlich vager und viel schwerer den einzelnen Individuen der Gesellschaft wie die in der Familie zuzuordnen.

Von daher verbietet sich die funktionalistische Vorgehensweise des Bundesverfassungsgerichts, über Beitragsdifferenzierung in der Pflegeversicherung familiale Leistungen für die Gesellschaft zu honorieren. Anstelle der Beitragsdifferenzierung bietet es sich an, über das Steuer- und Transfersystem einen gerechten Familienleistungsausgleich zu schaffen. Zentraler Ansatzpunkt muß dabei die »strukturelle Rücksichtslosigkeit (Fünfter Familienbericht $(1994,46)$ ) « sein. Diese finden wir nicht nur in der Pflegeversicherung, sondern in allen Lebensbereichen, so daß ein umfassender Ansatz gewählt werden muß, um den Art. 3 Abs. I und Art. 6 Abs. I GG gerecht zu werden.

Für eine Lösung über einen reformierten Familienleistungsausgleich spricht auch ein Gerechtigkeitsaspekt, auf den u. a. auch Ruland (200I, I676) hinweist. Da in der gesetzlichen Krankenversicherung und damit auch in der sozialen Pflegeversicherung oberhalb der Beitragsbemessungsgrenze, die auch gleichzeitig die Versicherungspflichtgrenze ist, Arbeitnehmer zwischen gesetzlicher und privater Versicherung wählen können, besteht die Gefahr, daß die reichen Kinderlosen aus der sozialen Pflegeversicherung aussteigen. Bei diesem Prozeß der adversen Selektion der »guten « finanzkräftigen Risiken kommt es dann dazu, daß in erster Linie die kinderreichen Mitglieder mit niedrigem und mittlerem Einkommen selbst über höhere Durchschnittsbeiträge eine Beitragsdifferenzierung finanzieren müssen. Im Extremfall unterstützen dann nicht die Kinderlosen, sondern die Mitglieder in der sozialen Pflegeversicherung mit wenigen diejenigen mit vielen Kindern. So führt die geforderte Beitragsdifferenzierung des Bundesverfassungsgerichts nicht zu einer Entlastung der kinderreichen Familien, sondern zu einer zusätzlichen Belastung, da die reichen Kinderlosen die Option zum Ausstieg aus der Solidargemeinschaft nutzen werden. So gesehen ist das Urteil des Bundesverfassungsgerichts den kinderreichen Familien nicht sehr dienlich. Für Ökonomen ist aber nicht die gute Absicht des Bundes- 
verfassungsgerichts, sondern das Ergebnis seines Urteils für eine Bewertung aus-

schlaggebend.

\section{Literaturverzeichnis}

Barro, R. J. (1974): Are Government Bonds Net Wealth?, Journal of Political Economy, Vol. 82, I095 - III 7 .

Borchert, J. (200I): Welche Folgen hat das Pflegeurteil für die Rentenversicherung?, Wirtschaftsdienst, 8I. Jg., $255^{-2} 59$.

BVerfG (200I a): Verfassungsmäßigkeit der privaten Pflegeversicherung (Urt. v. 3. 4. $200 \mathrm{I}$ - I BvR I68I/94 u. a.), Neue Juristische Wochenschrift, 54.Jg., I707 - 1709.

BVerfG (200I b): Bemessung des Beitrags zur sozialen Pflegeversicherung (Urt. v. 3. 4. $200 \mathrm{I}$ - I BvR I629/94), Neue Juristische Wochenschrift, 54.Jg., I712 - I716.

Burggraf, S. P. (1997): The feminine economy and enonomic man: Reviving the Role of Family in the Post-Industrial Age, Massachusetts, California, New York u.a.

Fünfter Familienbericht (1994): Familien und Familienpolitik im geeinten Deutschland Zukunft des Humanvermögens, Bonn.

Homburg, St./Gräff, C. (1988): Zur ökonomischen Begründbarkeit eines Familienlastenausgleichs, in: W. Albers und B. Felderer (Hrsg.), Familienlastenausgleich und demographische Entwicklung, Berlin, I $3-28$.

Kohli, M./Künemund, H./Motel, A./ Szydlik, M. (1999): Familiale Generationsbeziehungen im Wohlfahrtsstaat: Die Bedeutung privater intergenerationeller Hilfeleistungen und Transfers, WSI-Mitteilungen, 52.Jg., 20-25.

Lampert, H. (1996): Priorität für die Familie, Berlin.

Ott, N. (200I): Der Konstruktionsfehler im Sozialversicherungssystem bedarf einer Korrektur, Wirtschaftsdienst, 8I.Jg., 263-265.

Ribhegge, H. (199I): Allokative Aspekte der Kindererziehung - Zur Berücksichtigung der Kindererziehung in der Rentenversicherung und dem Familienlastenausgleich, List Forum, 17. Jg., S. 179-197.

Rürup, B. (200I): Ein Urteil mit begrenztem Gebrauchswert, Wirtschaftsdienst, 81. Jg., 259263.

Ruland, F. (200I): Das BVerfG und der Familienlastenausgleich in der Pflegeversicherung, Neue Juristische Wochenschrift, 54.Jg., I673-1744.

Sachverständigenrat zur Begutachtung der gesamtwirtschaftlichen Entwicklung (200I): Für Stetigkeit - gegen Aktionismus, Jahresgutachten 200I/02.

Schreiber, W. (1955): Existenzsicherheit in der industriellen Gesellschaft, Köln.

Sozialbeirat (200I): Stellungnahme des Sozialbeirats zu Urteilen des Bundesverfassungsgerichts zur Pflegeversicherung vom 3. April 200I hinsichtlich ihrer Bedeutung für die gesetzliche Rentenversicherung.

Weinberg, B. A. (200I): An Incentive Model of the Effect of Parental Income on Children, Journal of Political Economy, No. 2, Vol. 109, 266-279. 